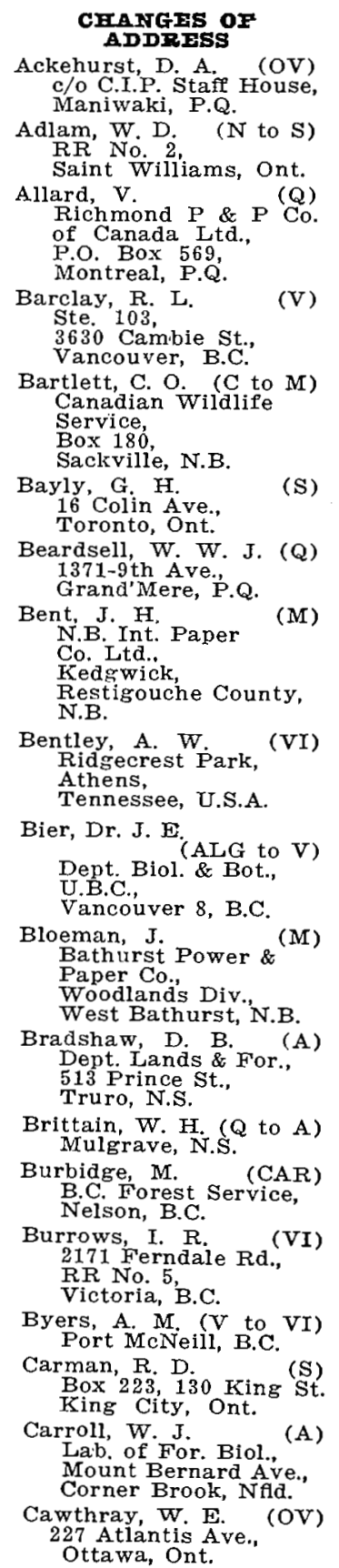

Champion, J. D. (RM) Box 226,
Hinton, Alta.

Chisholm, H. R. (VI) Ucluelet, B.C.

Cosen, G. G. Assistant Vice-Pres., Kimberley-Clark Corp.

isc., U.S.A.

Coyne, G. F. $(\mathrm{C}$ to $\mathrm{N}$ ) Dept. L. \& F.,

Cunningham, G. C. (M) For. Branch, Box 428 . Fredericton, N.B.

Dickson, R. B. (C to N) 280 Patricia Blvd.

Dickson, W. A.

P.O. Box H244,

Forestry Branch, Dept. N.A. \& N.R.

Dixon, R. V. (Q) LaCroche, Que., Via LaTuque.

Dow, A. W. Merton Lake 245 Quebec'street. Prince George, B.C.

Duffie, J. D. 838 Regent St Fredericton, N.B.

Dumont, A. C. General Delivery, Quesnel, B.C.

Dunne, A. N. Box 190, Blind River, "Ont

Eckel, L. H. (ALG to NW) Dept. L. \& F., Ont.

Edwards, R. W. Box 657 Terrace, B.C.

Elliott, R. J. P. (VI) B.C. Forest Service,
Forest Surveys Div., Fictoria, B.C

Engelhardt, N. T. (VI) For. Biology Lab., Victoria, B.C.

Farrar, Prof. J. Faculty of (ALG to S) Univ. of Toronto, Toronto, Ont.

Fellows, E. S. (RM to M) P.O. Box 354.
Fredericton, N.B.

Finan, D. J. Stevens, Ontario.
Foster, Dr. R. E. (VI) Dom. Lab, of For. 409 Fैederal Bldg.. Victoria, B.C.

Fowler, D. P. (U to S) Richmond Hill, Ont.

Fraser, R. C. (OV to N) P.O. Box 102,

Fries, J. W. St. Lawr. (Q) 840 Sun Life Blds.; Montreal, Que. 2220 Beach Drive (VI) Victoria, B.C.

Geoffrion, H. (SAG to $Q$ ) 931 Hainchaud Ave.,

Gibson, C. L. (C) Pineland Timber Co. Peter

Pntario.

Gimby, W. E. Ont. Dept. Lands Ti forests Toronto, Ontario

Gobeil, Dr. A. R. (Q) Quebec, P.Q. Godfrey, J. N.
2914 (V) Vancouver $8, B$.

Greaves, K. D. Anglo Can. P. \& P. Mills Ltd. Forestvile, P.Q.

Gustavson, E. S. Ste. No. 2 , 300 Montrose St.,
Port Alberni, B.C.

Hall, D. J. assell, T. T. (SAG) Consol. Paper Corp. Ltd., Roberval, Quebec.

Heaney, H. D. (M) Forestry Branch, Fredericton, N.B.

Hemmingsen, J. O (VI) $801-3$ rd Ave. N.,
Port Alberni, B.C.

Hepher, W. S. North Vancouver B.C.

Hess, Q. F. (N to $S$ ) Peterboro, Ontario

Holm, J. (VI) 798 Drake St..

Holmes, D. C.
Sarita River, B.C.

Hoogen, $A$. H. (OV to $Q$ ) 30808 F-O, R.C.A.F., Montreai, P.Q. 


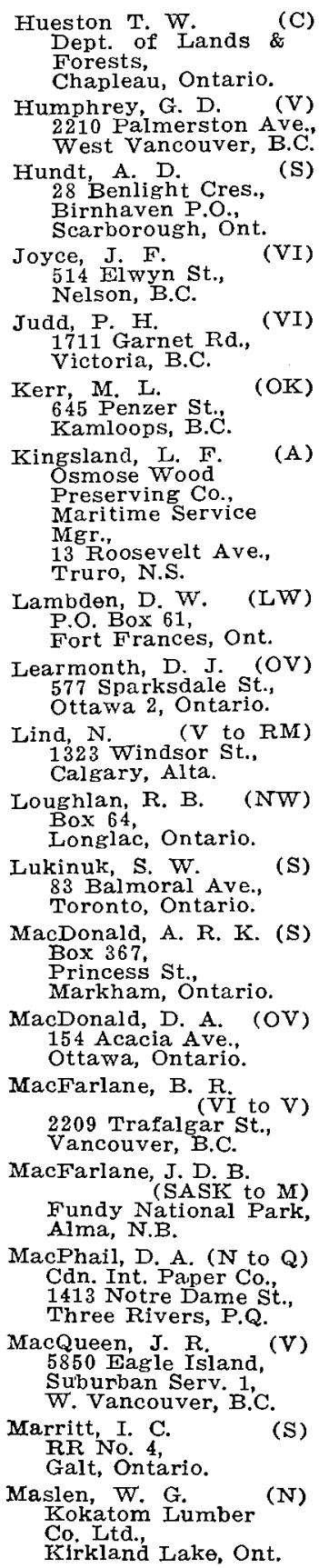

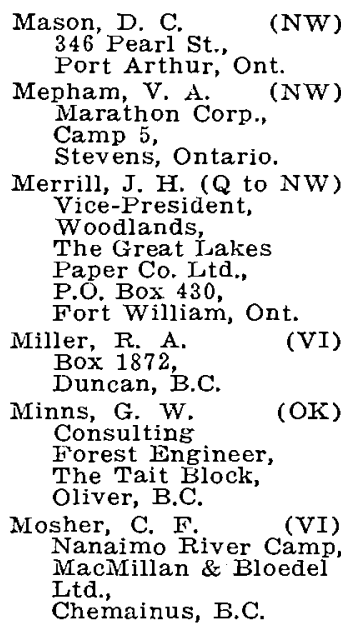

Miller, R. A. Dox 1872 , B.C.

Minns, G. W. Consulting Fiorest Engineer, The Tait Block, Oliver, B.C.

Mosher, C. F.

Nanaimo River (VI) MacMillan \& Bloedel Ltd., Chemainus, B.C.

Motyl, F. MT. ( $L W$ to $N W$ ) St. Lawrence Corp.

Beardmore, Ontario. Vancouver 8 , B.C.

Munro, D. W. (OK) 2212 Valleyview Dr., Kamloops, B.C.

Murray, F. T. (M to U) Woodlands Dept., Hammermill Paper Co.

Eire 6, Pa.

McDonald, C. I. D 202-19th St. (SASK) Prince Albert, Sask.

McGee, C. J. Wesmak Lumber We.stree, Ontario.

Moore, W. S. (Sag to Great Lakes Paper Co. Ltd., Fort William, Ontario Oosterhuis, H. T. (RM) Northwestern Pulp \& Power Ltd. Woodlands Div
Hinton, Alta.

Peacock, A. H. (S to C) Dept. Lands \& Forest

Porter, A. W. Massey Harris Ferguson Ltd., Market St Brantford," Ontario.

Ringham, $L$. ( $\mathrm{LW}$ to $\mathrm{N}$ ) Cochrane, Oñtario
Ruxton, J. 840 Eglinton Ave. W., Toronto, Ontario.

Shearsmith, G. V. (Q) Can. Int. Paper Co., Via LaTuque, P.Q.

Seed, G. K. ( . Co.

Camp Robinson, Ont.

Sewell, J. H. 180 Smythe (M) Fredericton, N.'B.

Sexsmith, I. R. (NW) Box 76 , Longlac, Ontario.

Sider, F. E. (N to LW) Dept. L. \& F.,

Sinclair, G. A. Ranser School, Dorset, Ontario

Sleeman, W. L. 2138 Victo (N to NW) Fort William, Ont.

Smith, D. W. H. J. Hodgins Ltd (VI) 625 Fort St.,

Smith, IE. W. (S to U) White Pine Sash Co., East 4005 Broadway, Spokane I0 Washington, U.S.A.

Smith, V. G. Ramsay, Ontario.

Snazelle, R. Bunbury Nursery, (A) RR No. 5, Charlottetown, P.E.I.

Switzer, A. L. K. (NW) Longlac, Ontario.

Thomas, I. (SAG to U) 18401 Hiller Ave., Ohio US 17

Todd, J. Oregon Saw Chain Ltd., 165 York Rd. Guelph, Ontario.

Toftdahl, $K$. 1756 Clow Rd., White Rock White Rock, B.C.

Topp, J. B. Box 782 ,
Sussex, N.B. (C) nderwood, T. 1169 East 14 th $\mathrm{St}$.
N. Vancouver, $\mathrm{B} . \mathrm{C}$. Van Fraassen, A M.

Pineland Timber Co. Ltd. Peterbell, Ontario

Waelti, H. (VI to CAR) Project 5059, c/o District Forester, Prince George, B.C. 
Walker, C. W. c/o C. D. Schultz $\&$ Co. $502-325$ Howe $\mathrm{St}$.

Walters, J. (OK to V) 21705 Ridgeway Haney, B.C.

Webb, L. S. 217 Roseberry St.,

Welsh, W. J. (V) T. \& H. Engineering \& Forestry Ltd. 207 W. Hastings St.

Whalen, J. M. (C to $\mathrm{S}$ ) Apt. 131, 51 Alexander St. Toronto 5, Ontario

Whelan, R. V. 32 Maryvale Cres (S) RR No. 1 Richmond Hill, Ont.

Wilde, C. J. R. ( $N$ to $S$ ) 357 Botsford St Newmarket, Ontario.

Wilson, D. M. (LW) Dryden, Ontario. Wilson, D. R. (C to $S$ )
Dept. Lands \& Forests,

Lindsay, Ontario.

Winters, E. 57 Rupert St (NW) Port Arthur, Ontario.

Wood, R. S P.O. Box 426

Wright, W $G$ $135 \mathrm{~W}$. Grande Allee, Quebec 6, P.Q. CORRECTION

Zacharkiw to Zachary,
M. Forest Survey Div., Victoria, B.C.

THESE MIFN AEI MITSING -

WHFRE ART THEY Allaire, R. H. H. (SAG)
Duhamel, P.Q. ton, S. $B$. Saint John, N.B.

Bridcut, W. C. Box 570, C.

Burke, $R$

Camp 5

(VI)

Bloedel, B.C.
Ekholm, K. E. 119 east st. Sault Ste. Marie, Ontario.

Fisher, R. B. 444 Arnold Ave.,

(C)

Girard, D. A. (VI) Ltd. Parson, B.C

Livland, J. G. 325 Howe St.,

Los, Otto, J. Espanoi Co. Ltd.,

MacMillan, C. J. Apt. 1, 1350 Prospect St., Sherbroolie, Quebec

Moore, F. R. (MAN) Man. For. Serv., For. Manasement Winnipeg, Manitoba

McAlister, E. P. 2506 Cedarwood Ave. Bellingham, Washington, U.S.A.

McInnes, D. L Box 393 Merritt, B.C.

Oldham, J. M. General Delivery, Vancouver, B.C.

Robinson, J. K. Fredericton, N.B.

Rushton, W. (CAR) General Delivery.

Rytz, A. A. (RM) Edmonton, Alta.

Shwed, Vitally Forest Div. P. S. C. 1450 O'Conner Dr. Toronto, Ontario.

Tessier, J. P. MacMillan \& Bloedel Co. Ltd., Sarita River Div., Port Alberni, B.C.

Thomson, T. M. (V) MacMillan \& Bloedel Co. Lta., Ash River Div.

Ast Alberni, B.C.

Weston, A. W. (VI)

Pioneer Timber Co.,
Port McNeill, B.C.

\section{NTW ACTIV}

Burton, D $\mathrm{H}$.

Southern Research

Station,

RR No. 2

ntario.

Dorland, J. T. Box 74

(NW) Longlac, Ontario.

Duffey, P. J. B. Petawawa Forest (V) Experiment Station Chalk River, Ontario.

Eades, H. W. (V) Forest Products Vancouver '8, B.C.

Mackay, K. M. (SAG) Dolbox 268

Poole, A. C. Box 152

Longlac, Ontario.

\section{NEW AFPITIATE}

NOBMBERS

Harrisson, F. S. (LW) Staff House,
Dryden Paper Co. Ltd., Dryden, Ontario.

MeCron, 1 . Longlac, Ontario.

Panczyszyn, W. A. (VI) B.C. Forest Products Ltd. Youbou, B.C.

\section{NFW STODENT \\ MEMESES}

University of Toronto

Wells, J. R

$361 \mathrm{Mt}$. Pleasant Rd. Toronto, Ontario.

RESIGNATIONS

Challenger, J. W. (V) 6537 Fremlin St.,

Haines, $H$. C. (U) S.T.I.C.A. Apartado, 1649 San Jose, Costa Rica

Joyce, J. F. (VI)

Warren, G. 5 Kimbourne Ave (S) Toronto, Ontario.

W)
W)
(I)
ts
o14 Elywn St.,

)

V)

AG)

